\title{
Multi-physics and multi-objective optimization of a high speed PMSM for high performance applications
}

\author{
Weiduo Zhao ${ }^{1}$, Xuejiao Wang ${ }^{2}$, Chris Gerada ${ }^{1,3}$, He Zhang $^{1}$, Chuan Liu ${ }^{1}$, and Yinli Wang ${ }^{1}$ \\ ${ }^{1}$ Faculty of Science and Engineering, University of Nottingham Ningbo China, Ningbo 315100, China \\ ${ }^{2}$ Servomotor BU, Yusheng Group, Ningbo 315100, China \\ ${ }^{3}$ Department of Electrical and Electronic Engineering, University of Nottingham, Nottingham NG7 2RD, U.K
}

\begin{abstract}
High-speed permanent magnet synchronous machines (PMSM) can operate with high power density and high efficiency which are often highly desirable in high performance applications. A multi-physics optimization program based on multi-objective genetic algorithm was developed in this paper, to achieve a tradeoff solution between the electromagnetic, mechanical and thermal aspects. $\mathrm{A}$ parametric electromagnetic model was modeled based on the finite element method (FEM) firstly, then a thermal network model and an analytical mechanical model to determine the thickness of the magnet and the sleeve were developed and merged within a design cycle of the machine, in an effort to attain the target performances of $20 \mathrm{~kW} / \mathrm{kg}$ at $20000 \mathrm{r} / \mathrm{min}$ for a 2 MW PMSM. Optimization results indicated that a final design with 8 poles and 48 slots could obtain a comprehensive performance between power density and efficiency.
\end{abstract}

Index Terms—High-speed PMSM, multi-physics, multi-objective, optimization.

\section{INTRODUCTION}

$\mathrm{H}_{u}$ IGH SPEED electrical machine has been developed and used for many years, and it is now considered a mature and reliable technology for a number of engineering applications, particularly for direct-drive solutions [1]. With the availability of high specification materials, development of power electronic converters and improvement in the manufacturing methodologies, there is currently an unprecedented effort towards developing electrical machines with very high power density and efficiency. This paper targets a power density of $20 \mathrm{~kW} / \mathrm{kg}$ for a $2 \mathrm{MW}$ drive running at $20 \mathrm{krpm}$, and the efficiency requirements need to be $>98 \%$. This requirement can meet a number of future potential requirements including marine and other transport system [2].

Based on a number of electrical machines surveyed, the $r p m \sqrt{ } k w$ was proposed as an index to demonstrate the level of difficulty in designing high speed machines [3]. Permanent magnet machines were identified as second best in achieving high peripheral speeds and best for power density. The target machine will reach the boundary for a PM machine with the $r p m \sqrt{ } k w$ of $8.94 \times 10^{5}$. Achieving these targets requires a multi-physics approach to the design, due to the interaction and conflict between the electromagnetic, mechanical and thermal aspects. Several researchers carried out research on this field, but most of them used an analytical optimization program [4][5], which may not obtain enough accuracy results especially for the electromagnetic analysis. Moreover, maximum power density and maximum efficiency can not be achieved together generally. There exists an optimal design to obtain a relative high performance for both power density and efficiency [6].

In this paper, a FEM based multi-physics and multiobjective optimization research is carried out to search for a

Manuscript received March 8, 2018. Corresponding author: Weiduo Zhao (e-mail: zhaoweiduo@gmail.com). best comprehensive performance between the power density and the efficiency. The multi-physical modeling approach and the key design issues are also described and discussed.

\section{DESIGN CONCEPT AND INITIAL CONSIDERATION}

According to the power and speed level, as well as the application background, several design concepts can be determined firstly on the basis of experience in previous work and references.

The surface-mounted permanent magnet (SPM) machine is selected as the main topology, due to higher allowable mechanical stress of rotor core than interior PM machine. The carbon fiber sleeve is used to fix the PM onto the rotor, since it has a great strength / density ratio. The magnets are cut into very small segments in the axial direction, to avoid high eddy current losses.

In order to provide opportunity for a high frequency design, some efforts from the materials point of view have also been adopted for the stator core and windings. Magnetic steel sheet of JNEX-900 $(0.1 \mathrm{~mm})$ with up to $6.5 \%$ of silicon content is selected due to its extremely low core loss. Rectangular transposed Litz wire is used to form the coils instead of the conventional wires, to reduce the AC losses, especially for the circulating currents between parallel strands during high frequency operation. Open slot is therefore adopted to let the rectangular preformed windings insert into the slot easily.

After selecting these concepts and methods, the number of design variables is reduced greatly, which will increase the optimization speed. Besides, the electromagnetic model is also simplified a lot, since 2D FEM is enough if eddy current losses on magnets are not considered. The complicated winding model is also not required, because the AC/DC loss ratio is nearly 1 by using transposed Litz wire with extremely small diameter. Therefore, it is possible to build a parametric electromagnetic model by 2D finite element method, as shown in Fig.1, with all the machine sizing variables inside the model. 


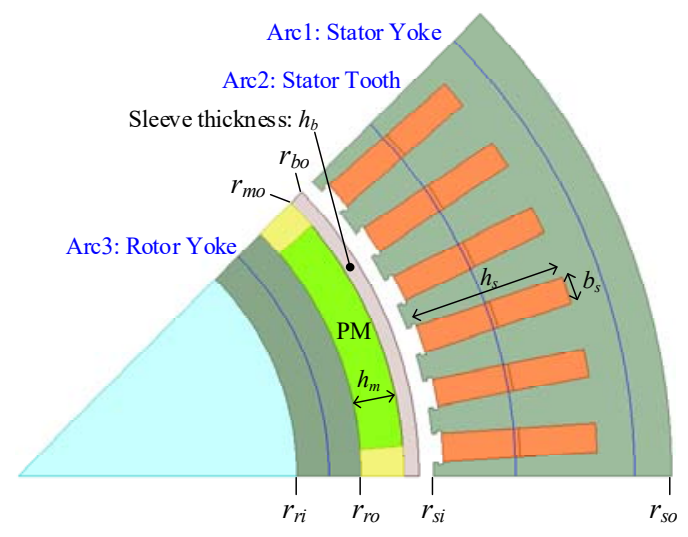

Fig. 1. Parametric finite element electromagnetic model.

\section{MUlti-Physics OPTIMIZATION}

\section{A. Design Variables}

The optimization can be divided into two levels. The number of poles is the upper level variable, while the machine dimensions are the lower level variables.

It is well known that increasing the number of poles will decrease the thickness of back iron on both rotor and stator, thus increasing the power density. However, the iron core losses will also increase due to a higher operation frequency. The copper losses may also increase in some degree, since the flux density in the air gap will reduced more with the increasing of number of poles. Therefore, there exists an optimal number of poles to obtain a relative high performance for both power density and efficiency. Considering the power and speed, as well as the limit of switching frequency, the number of poles are limited to $4 \sim 12$, and the number of slots are limited to $36 \sim 54$ with distributed windings.

Eight variables are selected to determine the machine sizing, as shown in Fig.1, which are stator outer and inner radius $r_{s o}$ and $r_{s i}$, rotor inner radius $r_{r i}$, active core length $L$, thickness of the sleeve and permanent magnet $h_{b}$ and $h_{m}$, and slot width and height $b_{s}$ and $h_{s}$. Some parameters are fixed to reduce the optimization dimension. The air gap thickness is fixed to 2 $\mathrm{mm}$, and the permanent magnet span is fixed to $144^{\circ}$, which are reasonable for a surface-mounted PM machine with such speed. Then the other dimension parameters can be calculated by these eight variables, as well as some output parameters, such as the motor mass and conductor area.

The stator inner radius should be lower than $97 \mathrm{~mm}$ to make sure the rotor linear speed is less than $200 \mathrm{~m} / \mathrm{s}$, which is the limit of silicon steel laminations. Several parameter relationships are also required to generate a motor successfully without any intersections, such as the stator outer radius should be larger than the sum of the stator inner radius and slot height.

For each slot and pole combination, the best design solution can be given by the machine sizing level optimization according to the certain objective. Then, the final optimized result can be obtained by comparing performances between each best solution.

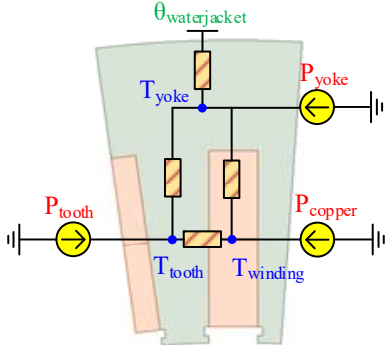

Fig. 2. Simplified thermal network model.

\section{B. Electromagnetic Modeling}

A 2D finite element model was built on the platform of ANSYS Electronics Desktop Maxwell. All the dimension parameters were modeled as variables when drawing the geometry of the machine. Eight dimension parameters mentioned before and phase current are selected as the input variables under the DesignXplorer setup. All the current is on the q-axis due to the MTPA control for the SPM motor. Several output variables we concerned are also defined and selected there, in order to let the optimization program call them and evaluate the performance. When defining the output parameters, time invariant variables can be obtained by employing methods detailed below even though transient simulation is performed.

1. Output power is defined as the total average shaft power by using IronPython scripting under User Define Output setup.

2. Core losses are defined as the iron core losses for both rotor and stator at the last simulation time, since the hysteresis losses need some time to develop, and the simulation time should be long enough ( $\geq 1.5$ electrical period).

3. Copper losses can be calculated with the input current, core length and slot area. A fill factor of 0.43 is used to define the ratio of copper area to slot area.

4. The flux density of the rotor yoke, stator tooth and yoke are also defined as the output variables. Middle lines of these three parts are drawn parametrically, and the maximum flux density on these lines at the last simulation time are defined as the output variables.

The other output variables are easier to be defined, such as the machine mass, current density, power density, torque per loss, and torque per loss per mass. These variables can be used as both the objectives and constrains

\section{Thermal Modeling}

Thermal issues are more important in high speed electrical machines with high power density. It is a combined electromagnetic and thermal coupling analysis to achieve an optimization design by maximizing the power density while limiting the temperature of key parts to their maximum allowed values. Therefore, a thermal model must be developed and integrated into the electromagnetic optimization program.

In this paper, a simplified thermal network of one slot span angle of the stator is developed as shown in Fig.2. Heat is assumed to flow radially outwards from the stator to a waterjacket, and the loss generation and heat dissipation of the rotor are neglected [7]. 


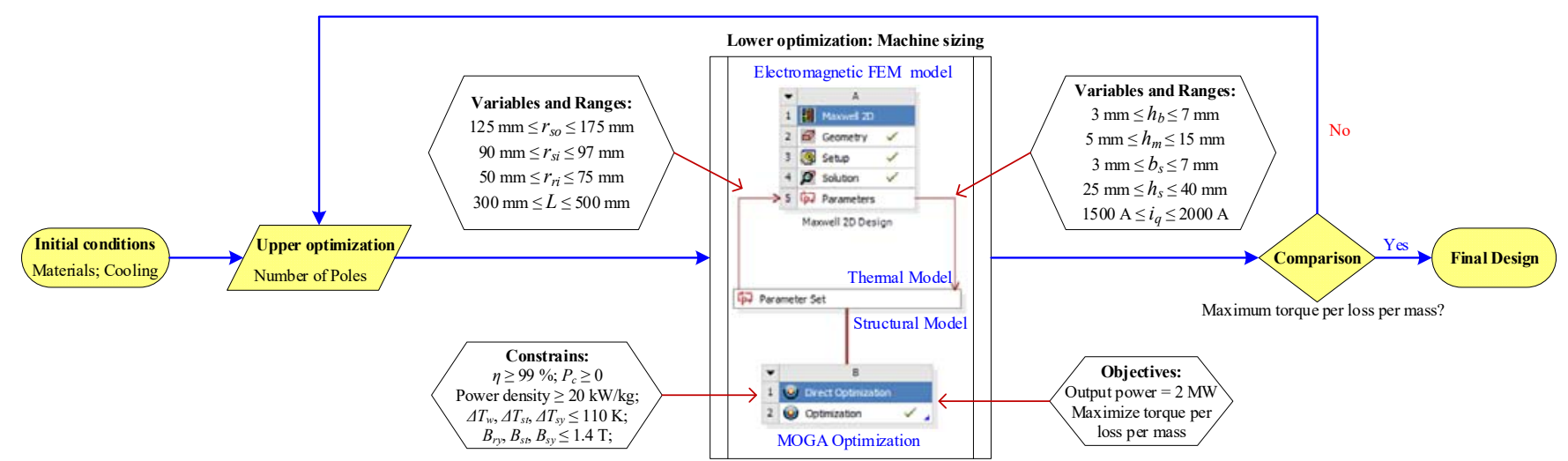

Fig. 3. Illustration of the optimization process.

The thermal network contains three nodes representing the temperature rise of the center of slot, tooth and yoke. The temperature of the water-jacket is considered as the reference temperature. Four thermal resistances between nodes and reference point represent the ability of heat conduction. Three power losses are considered as the corresponding heat sources and shown in the thermal network. The required data for calculating the network elements can be obtained from the input and output parameters of the electromagnetic simulation.

Based on the Kirchhoff's law, the equations of the thermal network can be obtained. Then the expressions of the temperature rise of these nodes can be analytically solved by using the mathematical software's symbolic operation function of matrix inversion, such as MathCad. The expression of the thermal resistance and the temperature rise can be written as the output parameters in Ansys workbench Parameter Set window. When a new design point is requested by the main optimization procedure, a FEM electromagnetic simulation is solved firstly, then temperature rise results can be obtained according to the machine sizing and electromagnetic performance, and used as constrains from the thermal point of view.

\section{Mechanical Stress Modeling}

A prefabricated carbon fiber sleeve embedded within an epoxy resin matrix is designed to ensure enough contact force to fix the magnets onto the rotor. The thickness of the sleeve $h_{b}$ is a key parameter for both electromagnetic and mechanical stress design, since it not only determines the thickness and position of the permanent magnet, but also determines thickness of the effective air gap. Therefore, an analytical mechanical stress modeling was carried out in this paper, to provide constrains for the main optimization program, and find a tradeoff design between electromagnetic and structure.

Using Young's modulus $E$, the sleeve undersize $\Delta D$, and other dimension parameters shown in Fig.1, the prestress and the contact pressure due to interference fit of the sleeve onto the magnets is given by [8]

$$
\begin{gathered}
\sigma_{t p s}=\frac{\Delta D}{2 \cdot r_{b}} E \\
p_{c p s}=\sigma_{t p s} \frac{r_{b o}^{2}-r_{m o}^{2}}{r_{b o}^{2}+r_{m o}^{2}}
\end{gathered}
$$

The rotational tangential stress of the sleeve (considered as thick shell) at speed $\omega$ is given by [9]

$$
\sigma_{t \omega s}=\frac{3-2 v}{8 \cdot(1-v)} \rho_{s} \omega^{2}\left(2 r_{b o}^{2}+\frac{2 \cdot(1-2 v)}{3-2 v} r_{m o}^{2}\right)
$$

where $v$ and $\rho_{s}$ are the Poisson ratio and density of the carbon fiber.

The stress inside the sleeve should less than the maximum permissible tangential stress $\sigma_{\text {tmax }}$, as given by (4), and then the undersize $\Delta D$ can be determined firstly.

$$
\sigma_{t}=\sigma_{t p s}+\sigma_{t \omega s}<\sigma_{t \max }
$$

The centrifugal forces on magnets and sleeve each reduce the total contact pressure between sleeve and magnets by

$$
p_{\omega s}=r_{b} \rho_{s} \omega^{2} h_{b} \text { and } p_{\omega m}=r_{m} \rho_{m} \omega^{2} h_{m}
$$

Where $r_{b}=\left(r_{b o}+r_{m o}\right) / 2, r_{m}=\left(r_{m o}+r_{r o}\right) / 2$.

Since the permanent magnet can sustain relative higher pressure but quite smaller tension, the sleeve must provide a positive residual contact pressure $P_{c}$ between the magnets and the rotor iron. Then constrains can be given by

$$
p_{c}=p_{c p s}-p_{\omega s}-p_{\omega m}>0
$$

The expression of these stresses and judgement equation can be written into the Parameter Set window, and used as the constrains from the structure point of view.

\section{E. Global Optimization Constrains and Objectives}

The electromagnetic, structure and thermal models mentioned above are integrated into the main optimization program, based on the ANSYS Workbench platform. The optimization process is shown in Fig.3. After a selection of the slot and pole combination manually in the first step, the second step is the multi-physics optimization to search for the best solution of machine sizing under certain constrains and objectives.

Constrains used in the multi-physics optimization include:

1. Flux density at the rotor yoke, stator tooth and yoke $B_{r y}$, $B_{s t}$ and $B_{s y} \leq 1.4 \mathrm{~T}$ (The saturation flux density of JNEX 900 is relative lower);

2. The residual contact pressure between the magnets and the rotor iron should be positive $P_{c} \geq 0 \mathrm{Mpa}$;

3. Maximum temperature rise of the winding, stator tooth and yoke $\Delta T_{w}, \Delta T_{s t}, \Delta T_{s y} \leq 110 \mathrm{~K}$ (The temperature of waterjacket is assumed to be constant of $70{ }^{\circ} \mathrm{C}$ ); 


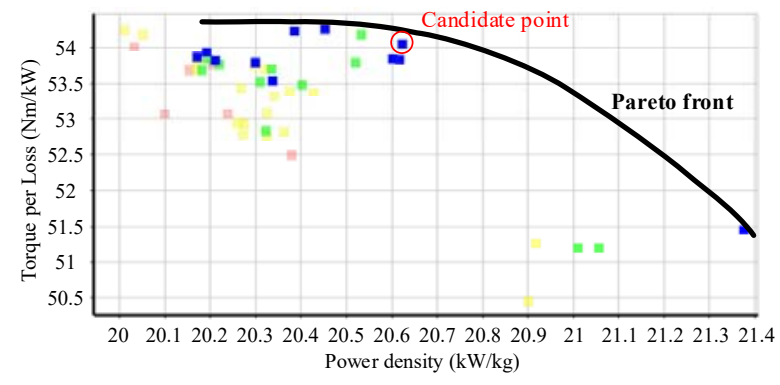

Fig. 4. Tradeoff plot of the optimization results.

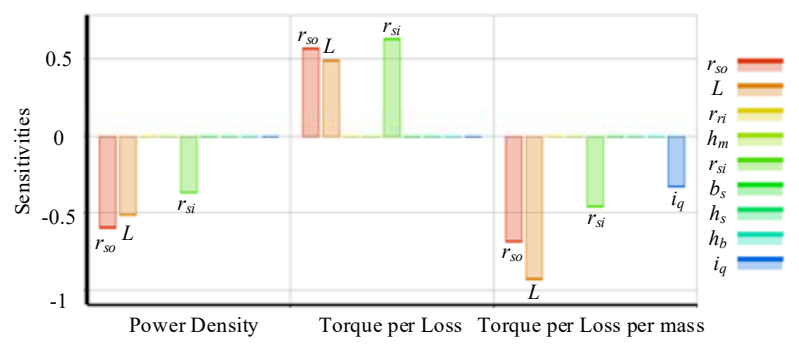

Fig. 5. Sensitivity plot of the optimization results.

4. Power density $\geq 20 \mathrm{~kW} / \mathrm{kg}$ (Active part including stator and rotor iron, stator copper and permanent magnets);

5. Efficiency $\eta \geq 99 \%$.

Multi-objective used in the optimization include:

1. Output power seeks target of $2 \mathrm{MW}$;

2. Maximize the torque per loss per mass $(\mathrm{Nm} /(\mathrm{kw} \times \mathrm{kg}))$ to obtain high performance of both power density and efficiency.

A MOGA method (Multi-objective Genetic Algorithm) is adopted for the optimization process. It is a variant of the popular NSGA-II (Non-dominated Sorted Genetic AlgorithmII) based on controlled elitism concepts [10]. It supports multiple objectives and constrains and aims at finding global optimum, therefore very suitable for the research in this paper.

\section{OPTIMIZATION RESULTS AND DISCUSSIONS}

\section{A. Optimization Tradeoffs}

Take the design of 8 poles and 48 slots as the example, after 693 evaluations (generated 12 offspring populations), the optimization converged. The total optimization time is about 6.5 hours for a 64-bit computer of Inter I7 3.6 GHz CPU with 16 GB RAM. A tradeoff plot can be obtained as shown in Fig.4, to show the improvement in the goal of power density can be achieved without sacrificing the torque per loss (efficiency).

In Fig.4, the black line represents the optimal limit (called Pareto front) that can be achieved under constrains defined in the optimization program. The "best" designs are those located on the Pareto front or closest to it. Four Pareto fronts are obtained in this optimization, and the best to worst go from blue to red respectively. The inverse hyperbola shape of the front clearly shows the power density and the efficiency are conflicting, which means a reduction in mass is generally associated with an efficiency decrease, and vice versa.

\section{B. Sensitivity Analysis}

Global Sensitivity chart shows sensitivity of each of the

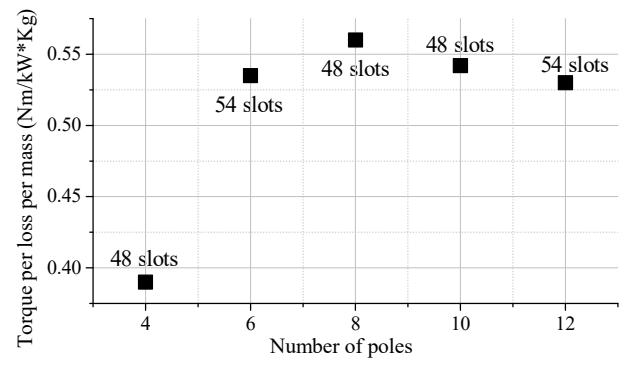

Fig. 6. Optimization candidates comparisons of different number of poles.

TABLE I

INPUT AND OUTPUT PARAMETERS OF THE CANDIDATE POINT

\begin{tabular}{cccc}
\hline \hline \multicolumn{2}{c}{ Input parameters } & \multicolumn{2}{c}{ Output parameters } \\
\hline$r_{s o}[\mathrm{~mm}]$ & 136.92 & $B_{r y}[\mathrm{~T}]$ & 1.24 \\
$r_{s i}[\mathrm{~mm}]$ & 92.65 & $B_{s t}[\mathrm{~T}]$ & 1.38 \\
$r_{r i}[\mathrm{~mm}]$ & 56.56 & $B_{s y}[\mathrm{~T}]$ & 1.375 \\
$L[\mathrm{~mm}]$ & 352.96 & $P_{c}[\mathrm{Mpa}]$ & 2.8 \\
$h_{b}[\mathrm{~mm}]$ & 5.45 & $\Delta T_{w}[\mathrm{~K}]$ & 105.7 \\
$h_{m}[\mathrm{~mm}]$ & 12.03 & $\Delta T_{s t}[\mathrm{~K}]$ & 79.36 \\
$b_{s}[\mathrm{~mm}]$ & 6.2 & $\Delta T_{s y}[\mathrm{~K}]$ & 16.28 \\
$h_{s}[\mathrm{~mm}]$ & 27.22 & $\eta[\%]$ & 99.13 \\
$i_{q}[\mathrm{~A}]$ & 1902 & Power Density $[\mathrm{kW} / \mathrm{kg}]$ & 20.62 \\
\hline \hline
\end{tabular}

output parameters with respect to input variables, as shown in Fig.5. It can be seen clearly that the stator outer and inner radius $r_{s o}$ and $r_{s i}$ (or split ratio), and active core length $L$ are the key parameters to the optimization objectives. The other five machine dimension parameters should be limited by the constrains and be closely correlated with these three key parameters. The output parameters of power density and torque per loss per mass have a negative sensitivity with $r_{s o}, r_{s i}$ and $L$, while the torque per loss has a positive sensitivity with them. The parameter of torque per loss per mass is also negative sensitive with the max phase current $i_{q}$. All these sensitivity trends are consistent with common experience of machine design, moreover, the proportion of importance can also be obtained from the chart.

\section{Candidate Point and Performance}

After several rounds multi-physics and multi-objectives optimizations, the global candidates' comparisons of optimal torque per loss per mass solution with different number of poles are shown in Fig.6. It shows the 8 poles with 48 slots design can obtain a best comprehensive performance between the power density and the efficiency, with the torque per loss per mass of $0.56 \mathrm{Nm} /(\mathrm{kW} \times \mathrm{kg})$ and power of $2 \mathrm{MW}$. The efficiency of the candidate is $99.13 \%$, with the copper loss of $11862 \mathrm{~W}$ and iron loss of $5720.5 \mathrm{~W}$. The parameters and the performance of the final candidate are listed in Table. I. It can be seen that the final design can meet all the requirements from electromagnetic, structural and thermal point of view.

\section{CONCLUSION}

In this paper, a multi-objective optimization research is carried out based on a multi-physical program, including FEM electromagnetic, thermal network and analytical structural simulation. A torque per loss per mass parameter is used as the optimization objective to represent a comprehensive 
performance between the power density and the efficiency. The multi-physical modeling method and the key design issues are described and discussed in detail. According to the optimization and sensitivity analysis results, a final design of 8 poles and 48 slots with detail parameters listed in Table. I is selected, and the performance meets all the requirements.

\section{ACKNOWLEDGMENT}

This work was supported in part by the Zhejiang Natural Science Foundation under Grant LQ18E070003, in part by the Ningbo Natural Science Foundation under Grant 2017A610089 and in part by the Ningbo Science \& Technology Beauro under Grant 2013A31012 and 2014A35007.

\section{REFERENCES}

[1] A. Boglietti, C. Gerada, and A. Cavagnino, "High speed electrical machines and drives", IEEE Trans. Ind. Electron., vol. 61, no. 6, pp. 2943-2945, Jun. 2014.

[2] European Commission, "Flightpath 2050: Europe's vision for aviation", $<$ http://ec.europa.eu/transport/modes/air/doc/flightpath2050.pdf>, 2011.

[3] D. Gerada, et al., "High-speed electrical machines: Technologies trends and developments", IEEE Trans. Ind. Electron., vol. 61, no. 6, pp. 29462959, June 2014.

[4] H. Gorginpour, et al., "Electromagnetic-thermal design optimization of the brushless doubly fed induction generator", IEEE Trans. Ind. Electron., vol. 61, no. 4, pp. 1710-1721, Apr. 2014.

[5] X. Zhang, et al., "CQICO and multiobjective thermal optimization for high-speed PM generator", IEEE Trans. Magn., vol. 53, no. 6, p. 8201604, Jun. 2017

[6] Tenconi, S. Vaschetto, and A. Vigliani, "Electrical machines for highspeed applications: Design considerations and tradeoffs", IEEE Trans. Ind. Electron., vol. 61, no. 6, pp. 3022-3029, Jun. 2014.

[7] P. Arumugam, et al., "Design optimization of a short-term duty electrical machine for extreme environment", IEEE Trans. Ind. Electron., vol. 64, no. 12, pp. 9784-9794, Dec. 2017.

[8] Binder, T. Schneider, and M. Klohr, "Fixation of buried and surface mounted magnets in high-speed permanent magnet synchronous motors", Conf. Rec. 40th IEEE IAS Annu. Meeting, pp. 2843-2848, Oct. 2005.

[9] Z. Huang and J. Fang, "Multiphysics design and optimization of highspeed permanent-magnet electrical machines for air blower applications", IEEE Trans. Ind. Electron., vol. 63, no. 5, pp. 2766-2774, May 2016.

[10] Ansys workbench help, "Multi-Objective Genetic Algorithm (MOGA)" https://www.sharcnet.ca/Software/Ansys/17.0/enus/help/wb_dx/dxBEMtemp11.html 